علاقة الإيقاع الحيوي العقلي بالدفاع عن الملعب لدى اللاعب الحر بالكرة الطائرة

م.د. ازاد حسن قادر

كلية التربية الرياضية جامعة صلاح الدين

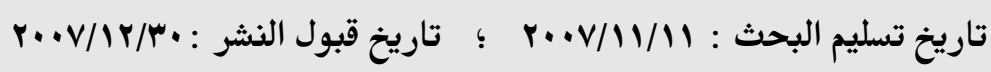

الملخص

هدف البحث الى

- التعرف على علاقة الايقاع الحيوي بالدفاع عن الملعب للدى اللاعب الحر بالكرة الطائرة

لفرق منتخبات الجامعات العراقية.

تكمن أهمية البحث في التعرف على علاقة الايقاع الحيوي ومنها تركيز الانتباه بالدفاع عن الهن

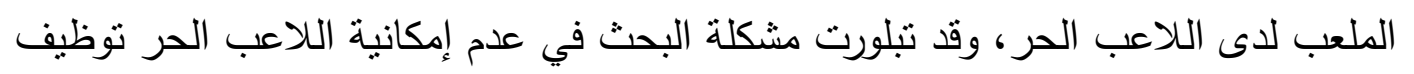

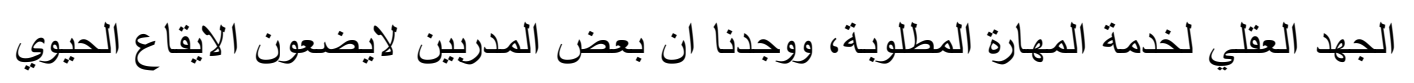
موضع التطبيق عند وضع خطتهم التنريبية والسباقات.

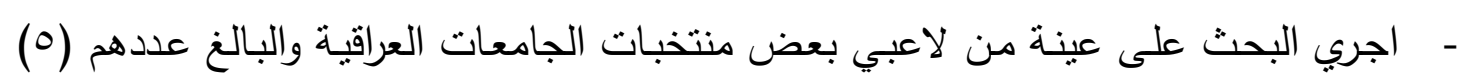

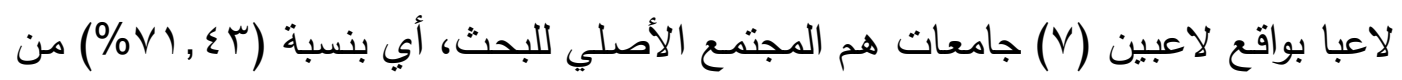

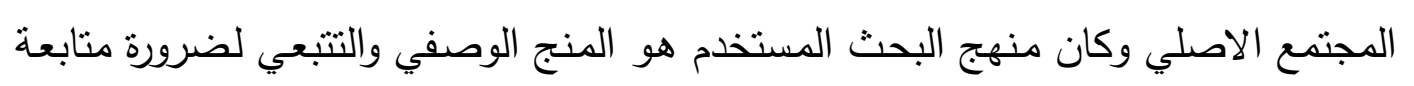

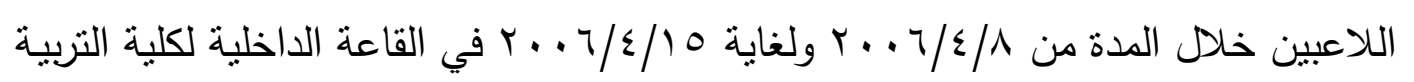

$$
\text { الرياضية في جامعة صلاح الدين - اربيل }
$$

- ويعد عرض النتائج وتحليلها ومناقشتها تم التوصل الى استنتاجات عدة اهمها

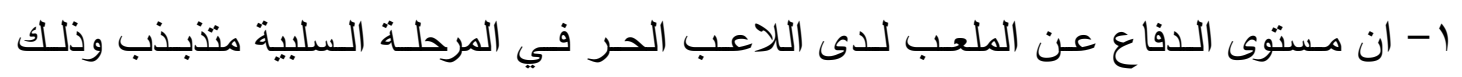

$$
\text { لاستعداده المتدني بعض الثيء في المستوى العقلي. }
$$

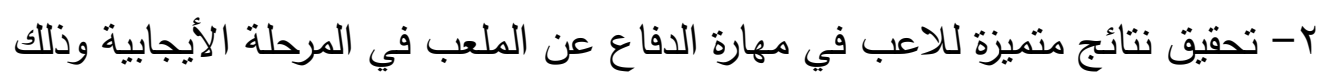

لأمتلاكه درجة عالية من تركيز الأنتباه. 


$$
\begin{aligned}
& \text { وقد قدم الباحث عدة التوصيات اهمها } \\
& \text { 1- ضرورة مراعاة اللاعبين في اوقات ومراحل الايقاع عند تحديد المنهج التدريبي و اجراء } \\
& \text { البابقات في دوري الجامعات. } \\
& \text { r- اعطلاء اللاعب الدافعيـة في المرحلـة الأيجابيـة اذ انها تؤودي الى زيـادة مستوى الأنجاز ، }
\end{aligned}
$$

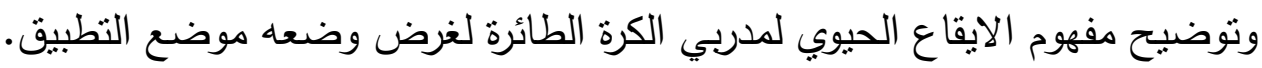

$$
\begin{aligned}
& \underline{\text { ABSTRACT }}
\end{aligned}
$$

The relation Ship between mental rhythmic Sycle and defending filed of free player in volleyball.

\author{
By : Dr.Azad .H. Qader
}

University of salahaddin /College of physical education

\title{
Important of the study:
}

The great concern in this study was to identify the relation ship between mental rhythmic sycle of which concentrating attention with defending on field of free player. The main problem of this study was, the free player did not have ability to function his mental efforts to serve the demanding skill. Also, some coaches did not put the vital rhythmic into consideration during competitions and trainning plans.

\section{Aim of the study:}

To identify the relation of rhythmic with defending on the field of free player for varsity volley ball of Iraqi universities.

\section{Methodology}

- A sample of university varsity players of volley ball were in this study (5)players of (7 universities) .

- The percentage of sample was (\%71.43) .

- A descriptive study was used in this study.

- The study was applied during the period of 8/4 - 15/4/2006

- This study was conducted in the university of Salahaddin - Arbil.

\section{Conclusion and Recommendations}

On the basis of the analysis of the data, the following conclusions were presented:

1- The level of field defense for free player at the negative stage was unstable, due to, Bad readiness in the mental level.

2- Excellent results have been a chieved for the player in field defense skill at positive stage, due to high degree of concentrating attention.

\section{The researcher recommended :}

1- Mor emphasis on scheduling stages of rhythmic should be taken into considerationWhen limiting trainning program and tournaments of universities. 
2- More emphasis on playing motivation at the positive stage should be taken Into consideration which will increase performance level and explanation of vital rhythmic concept for volley ball trainners into application.

\section{1 - التعريف بالبحث}

\section{1- 1 - 1 مقدمة البحث وأهميته}

يعد المجال الرياضي من اهم المجالات التي طبق فيهـ نظريات الإيقاع الحيوي وان

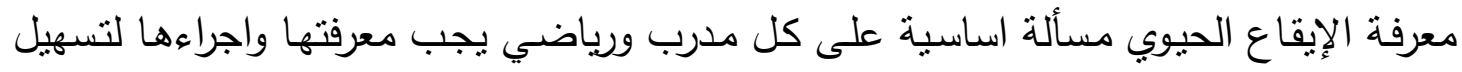
تتظيم الحياة اليومية للرياضي بحيث يكون ايقاعه الحيوي في اعلى مستوى له في الوقت المحدد

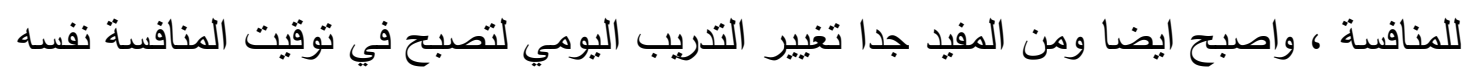

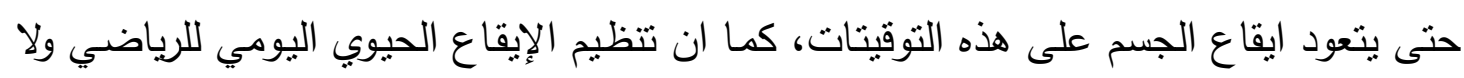

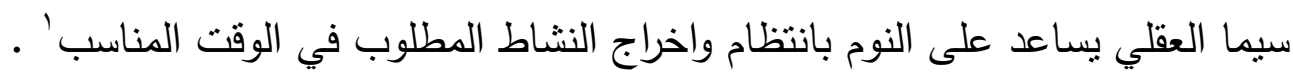

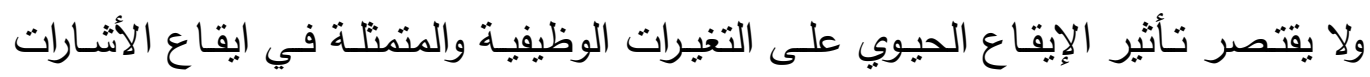

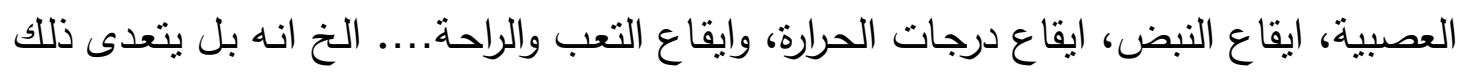

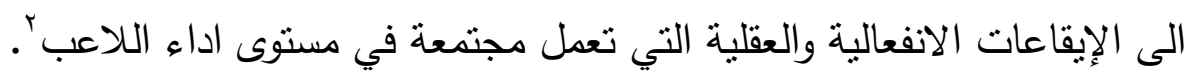

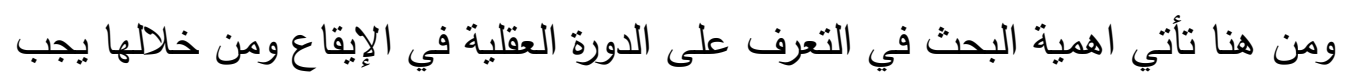

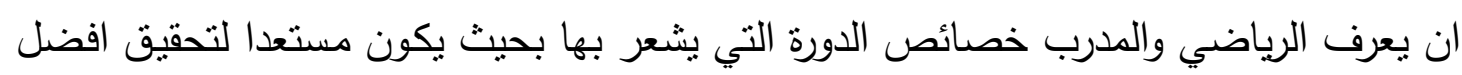
انجاز في الدفاع عن الملعب في اثثاء المنافسة.

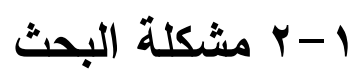

تطورت لعبة الكرة الطائرة تطوراً كبيراً ولاسيما خلال الحقبة الأخيرة من هذا القرن، واتسع البعاء

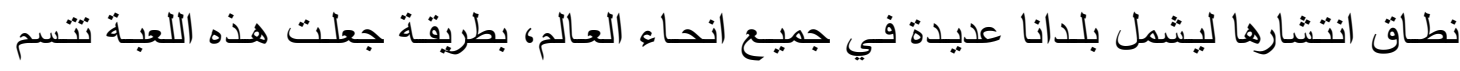

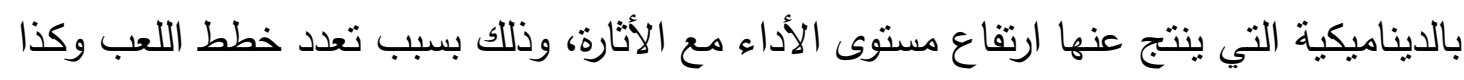

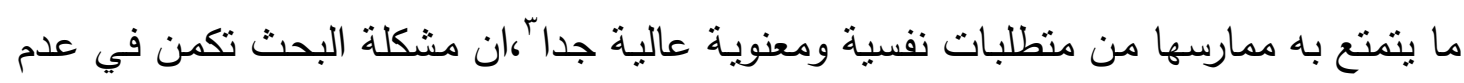
امكانية اللاعب الحر نوظيف الجهد العقلي لخدمة مهارة الدفاع عن الملعب من خلال انتباهيه

1

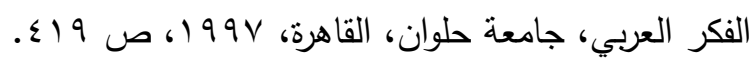

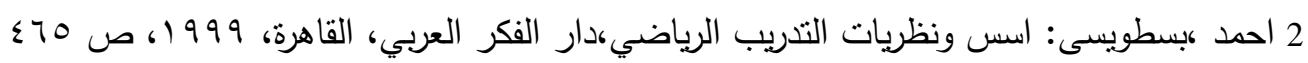

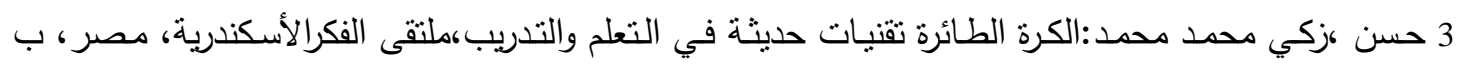


وتوقعه الحركي الجيد من خلال القدرة العقلية الكبيرة لتوقع مكان سقوط الكرة، وعدم اخذ بعض المدربين الإيقاع الحيوي بعين الأعتبار عند وضع خطنه العندم التدريبية . 1- r - البحث

- التعرف على العلاقة بين قيم الدورة العقلية الإيقاع الحيوي ممثلة (بتركيز الأنتباه) ومهارة الدفاع عن الملعب لدى اللاعب الحر بالكرة الطائرة.

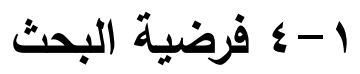

- - هناك ارتباط معنوي بين الإيقاع الحيوي العقلي( تركيز الأنتباه) ومهارة الدفاع عن الملعب لاى اللاعب الحر بالكرة الطائرة.

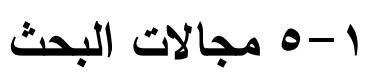

1-1-1 المجال البشري : لاعبو فرق منتخبات الجامعات العراقية بالكرة الطائرة .

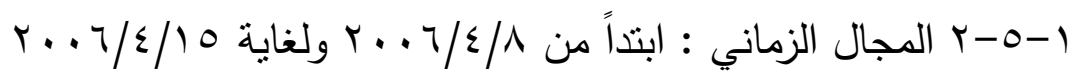

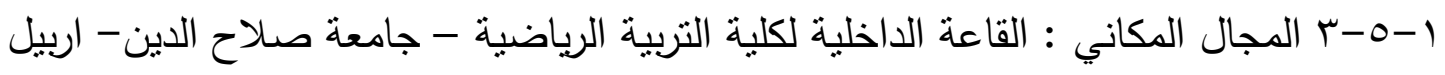
r r r r الاسات النظرية

$$
\text { r - إ مفهوم ومعنى الإيقاع الحيوي }
$$

اول من استخدم مفهوم ومصطلح الإيقاع الحيوي هم اليونانيون القدماء تحت مصطلح الإنح

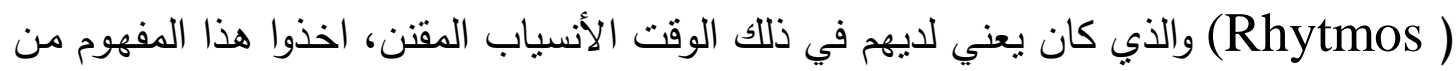

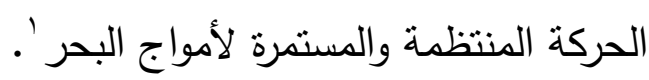
وعرفه "علي بيك ،وصبري عمر " بانه التغيرات الحيوية المنظظة ذات المدى القريب والبعيد

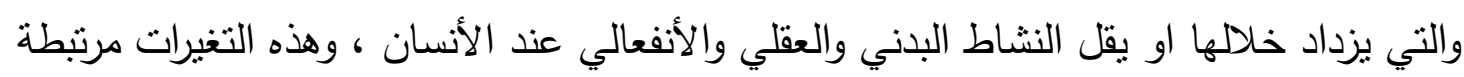

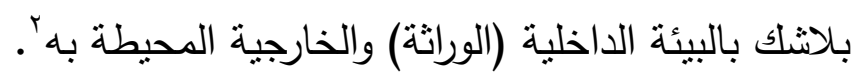

\section{r - بالإيقاع الحيوي وأهميته في الكرة الطائرة}

يلعب الإيقاع الحيوي دوراً فاعلاً في أداء فعاليات اللعب فيالكرة الطائرة الطائرة ويؤئر بالنشاط العقلي للفرد في لذاكرة واليقظة، والقدرة على التعلم، وسـلامة العمليـات العقليـة ، وان مفهوم

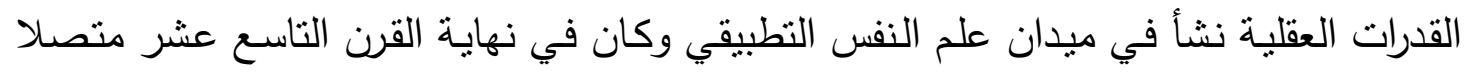

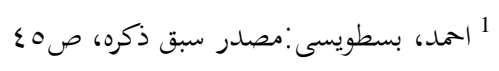

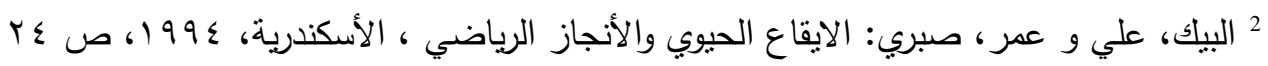


بالدراسات التجريبية وفي بداية القرن العشرين ظهر في فرنسا مرنبطا بقياس الذكاء في ابحاث العالم'

تعد القدرات العقلية التي يتميز بها الأنسان من بين اهم العوامل والمحددات في عملية تعلم

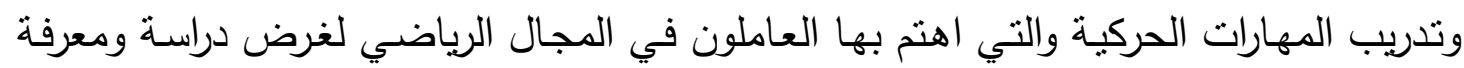
الفروق الفردية بين الأشخاص.

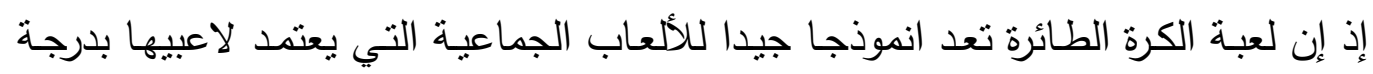

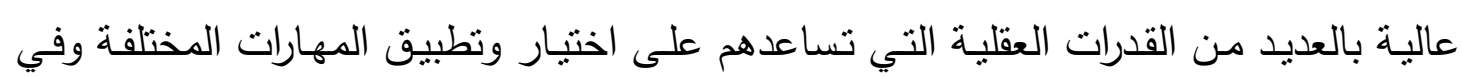
توقيت ادائها المناسب بمنتهى السرعة والدقة.

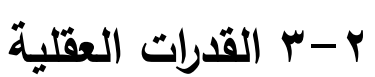

r-r

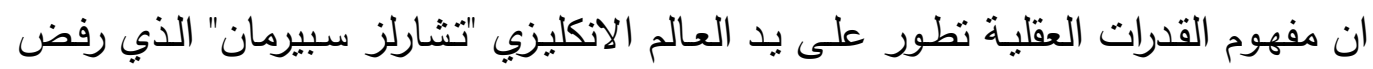

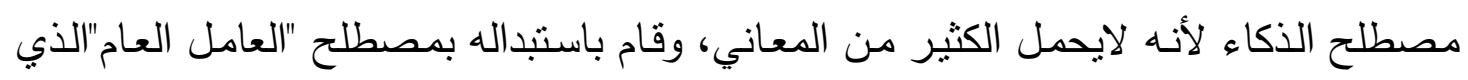

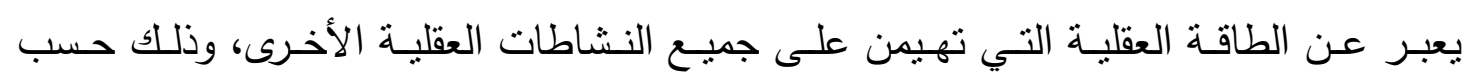
مقتضيات نظريته المعروفة (بنظريـة العاملين) ويتفق معظم علمـاء النفس على تعريف القدرة

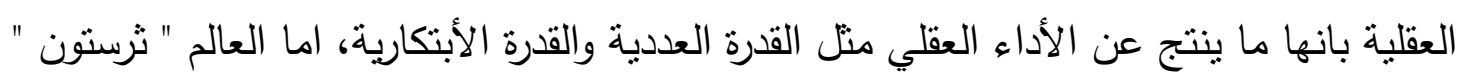

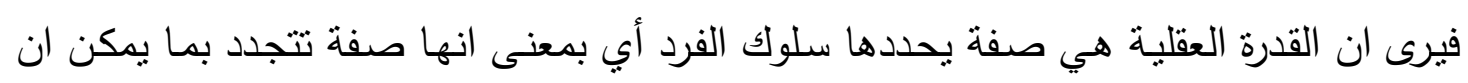

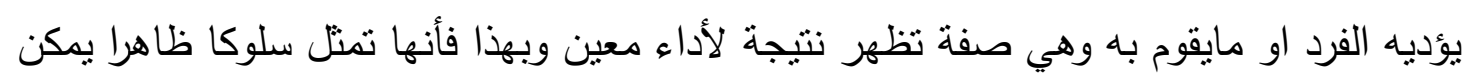

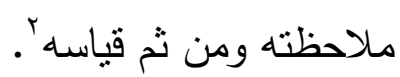
قد تتاول الباحث تركيز الأنتباه ممثلا للقدرات العقلية لقياس الدورة العقلية لذا سيتتاول

$$
\begin{aligned}
& \text { الباحث شرح الأنتباه في الدجال الرياضي: } \\
& \text { r }
\end{aligned}
$$

r r-r

يعد الأنتباه من العوامل المهوة للأداء الناجح للمهارات الرياضية، فالرياضي لاهيت يستطيع

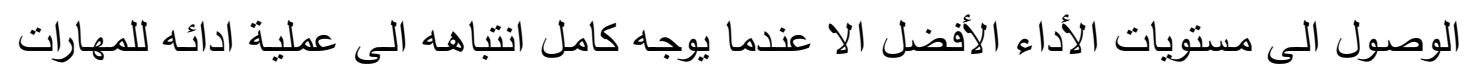

1 موسى ،عدنان هادي : علاقة بعض القدرات العقلية بفن الأداء المهاري ومستوى الأنجاز

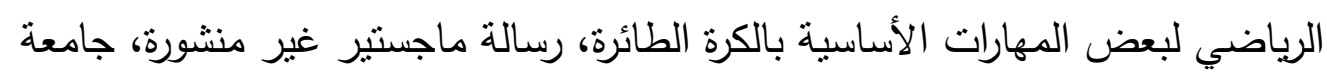

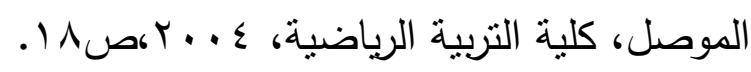

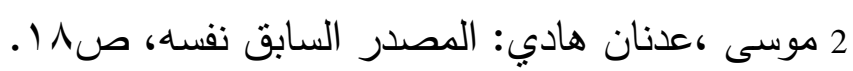


دون الأثشياء الأخرى، وان الأداء المتميز لايحدث الا عندما يركز الرياضي انتباهه على العوامل

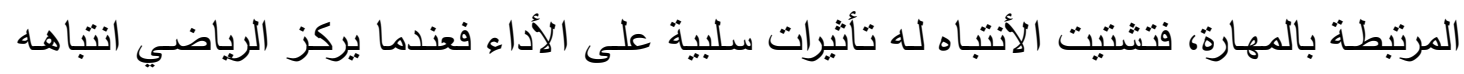

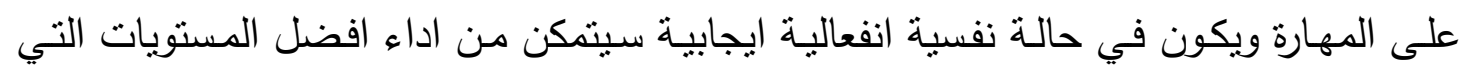

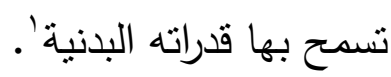

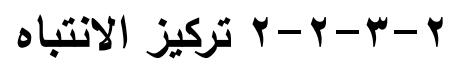

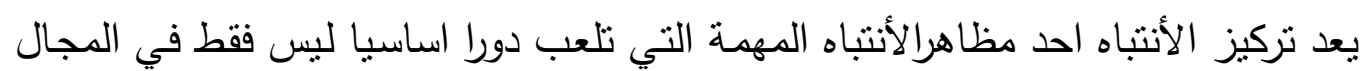

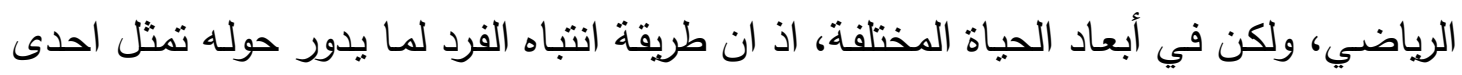

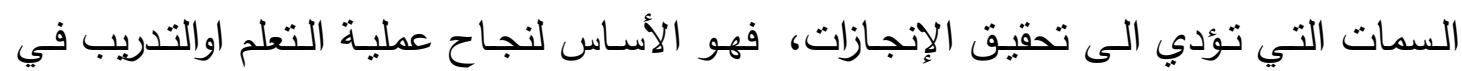

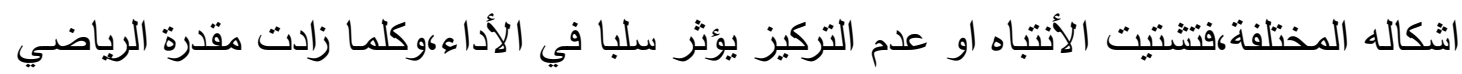

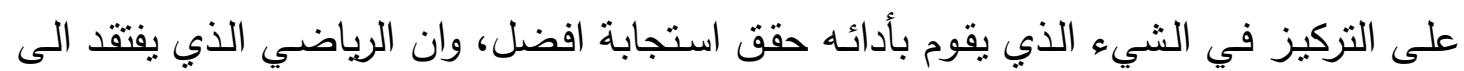

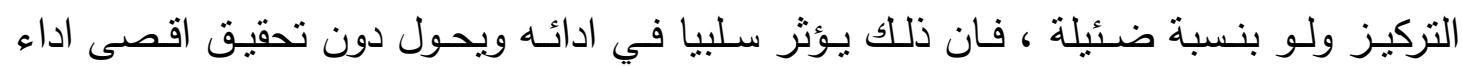
ممكن.

\section{r- منهج البحث واجراء|ته الميدانيه \\ r-1 1- 1 منهج البحث}

أن طبيعة المشكلة تلزم الباحث في اختيار المنهج الملائم، لذا أستخدم الباحث المنهج الوصفي لملائمته مشكلة البحث .

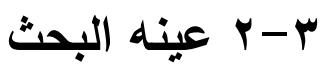

من الامور الواجبة في البحث العلمي اختيار عينة تمثل المجتمع الاصلي تمثيلاً صادقاً

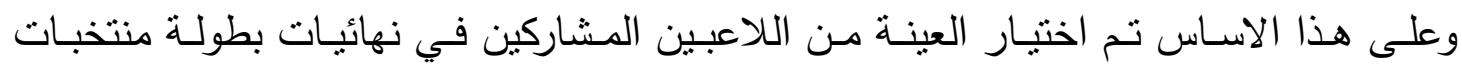

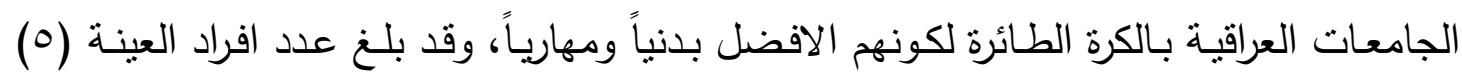

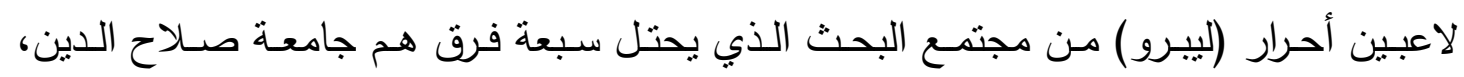
والسليمانية ، وديالى والموصل، ودهوك، وتكريت، وكركوك تم استبعاد جامعتي دهوك، وكركوك،

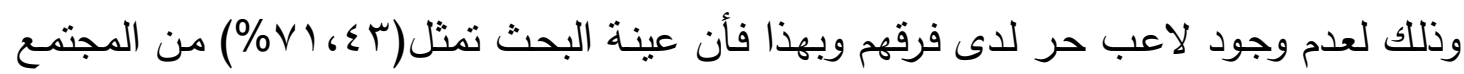
الاصلي.

وتعرف الباحث على منحنيـات الإيقاع الحيوي الحقيقي للاعب مـن خـلال الطريقة

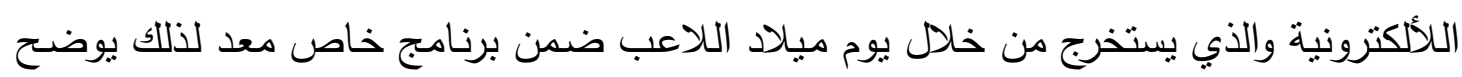
فيه دورات اللاعب ومراحل إيقاعه الحيوي.

1 1 النقيب، يحيى كاظم: علم النفس الرياضي ،معهد عداد القادة، مطابع الأيوبي، الرياض، السعودية، ـ99 (19.

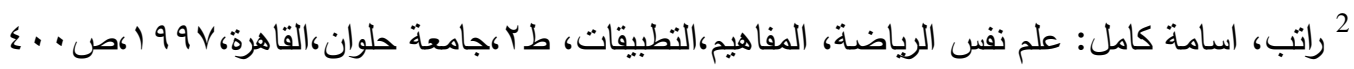


- الملاحظة العلمية من خلال استمارة خاصة لتقويم مهارة الدفاع عن الملعب (ملحق رقم ( ).

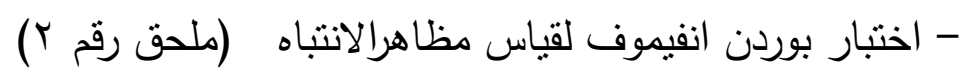
r- الأدوات المستخدمة في البحث: - جهاز الحاسوب الالكتروني

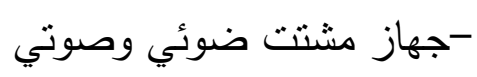
-كرات طائرة قانونية نوع(mikasa)

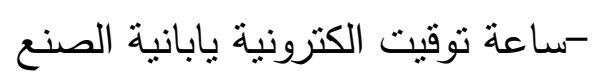

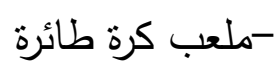
- ادوات مكتبية

r- ب-ه خوات تنفيذ البحث r- - الاختبارات المستخدمة في البحث r-ه - - - اختبار تقويم مهارة الدفاع عن الملعب .

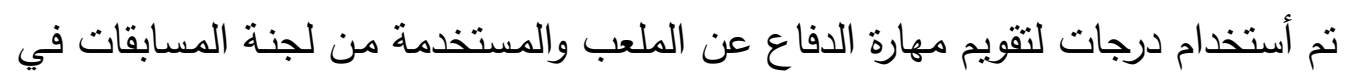
الاتحاد الدولي عند تقويمهم للمهارات في البطولات الدولية وكانت درجات الثقويم كالأتي ' - ثلات درجات للافاع الجيد الذي من جرائه يمكن عمل هجوم مثالي.

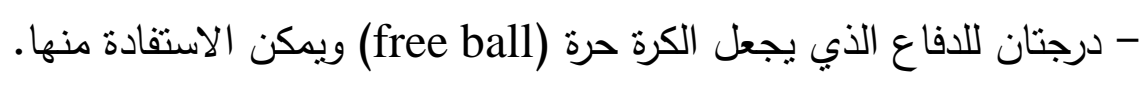

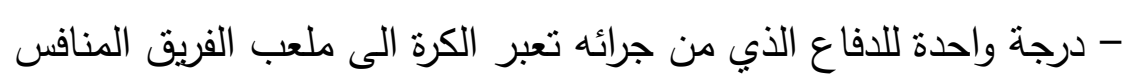

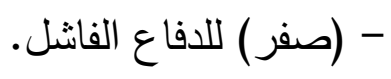

r-1- r- r- (ختبار تركيز الانتباه

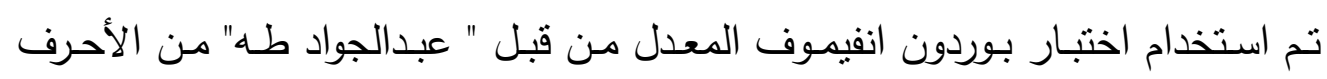
الروسية الى الأرقام العربية.

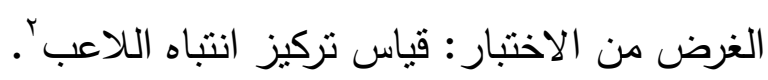

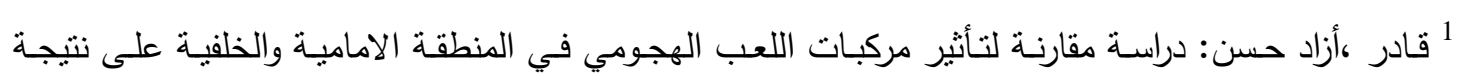

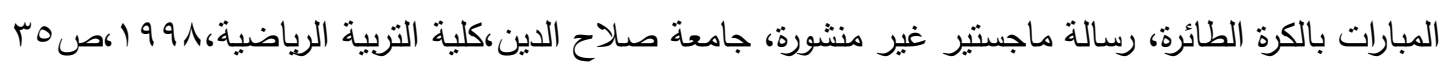
2² المصدر السابق ، عدنان هادي، ص >1. 
الأدوات : جهاز مشتت ضوئي وصوتي، ورقة قياس الأنتباه، ساعة التوقيت، قلم

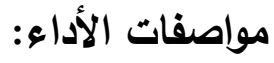

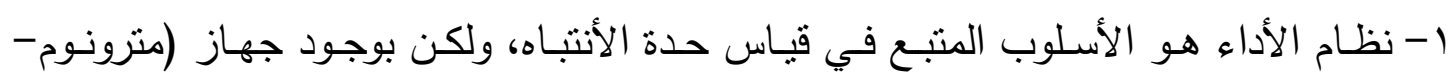

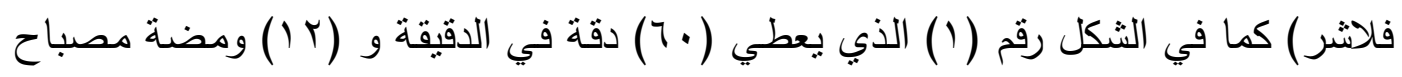

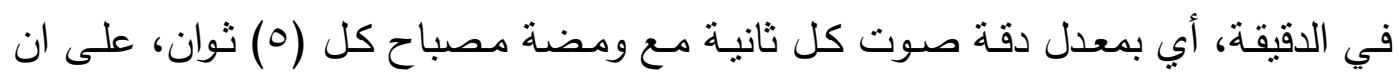

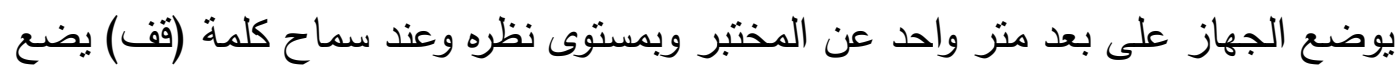
اللاعب علامة راسية عند اخر رقم يشطب او وصل اليه.

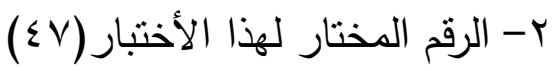

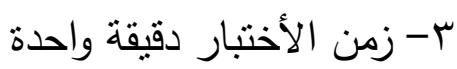

ع - احصاء العدد الكلي للأرقام التي وصل اليها اللاعب خلال دقيقة واحدة للأختبار حتى كلمة

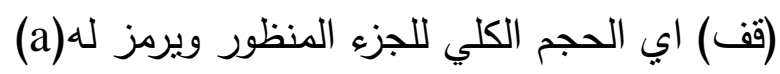

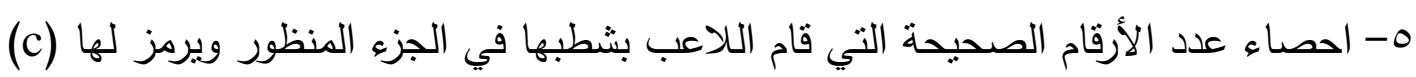
7- احصاء عدد الأرقام التي قام اللاعب بشطبها عن طريق الخطاء في الجزء المنظور اذ يرمز

(w)

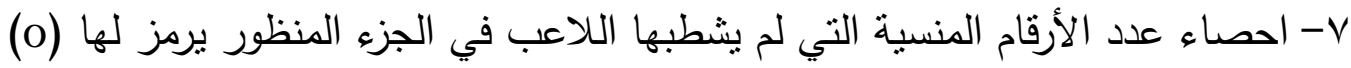

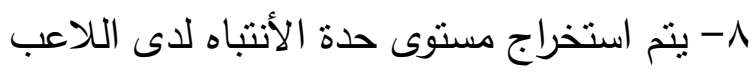
طريقة التصحيح وإحتساب النتائج 1- المعادلة التي استخدمت في حدة الأنتباه تستخدم في التركيز عدد الأرقام المشطوبة صحيحا - عدد الأرقام المشطوبة خطا حدة الأنتباه = _ـ عد الأرقام المنظورة عدد الأرقام المشطوبة صحيحا - عدد الأرقام المنسية الأنطوة

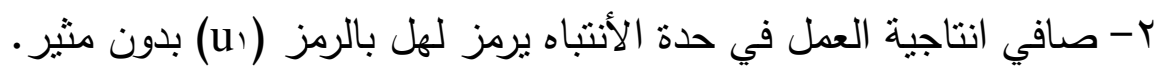

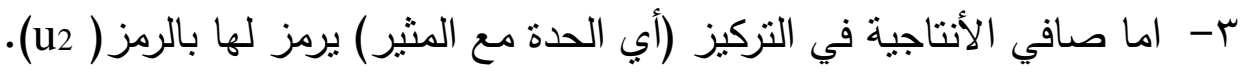
ع - تركيز الأنتباه يستخرج في النتيجة النهائية عن طريق المعادلة الأتية. تركيز الأنتباه = الأختبار بدون مثير - الأختبار مع المثير لئني $\mathrm{U} 2-\mathrm{U} 1$

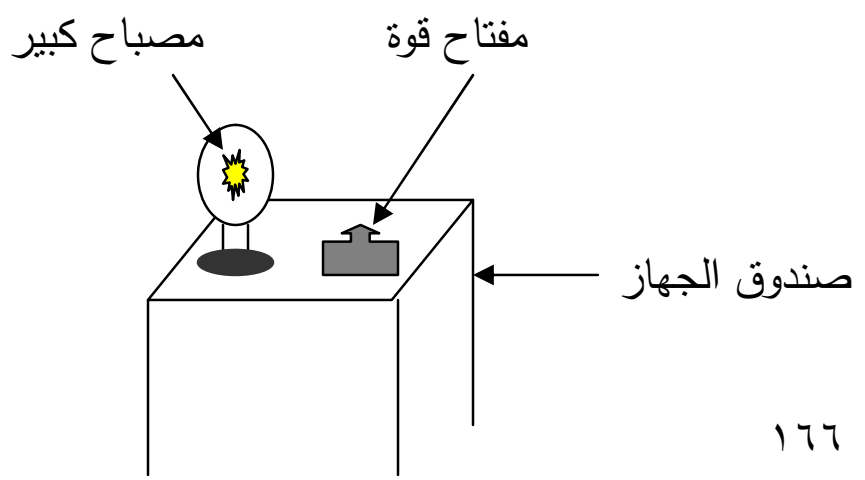




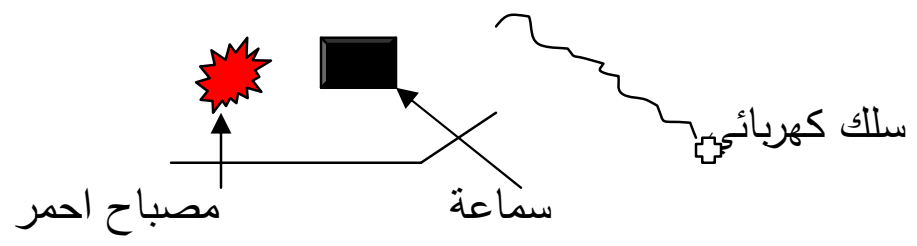

الثكل رقم (1)

يوضح جهاز المترونوم فلاشر لقياس تركيز الأنتباه

\author{
r- ( - الوسائل الأحصائية \\ -

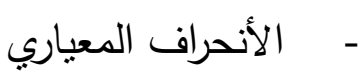 \\ - معامل الارتباط' -
}

ع -عرض ومناقشة النتائج

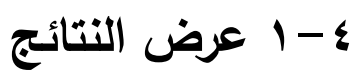

تم عرض النتائج ومناقشتها في ضوء البيانات الأحصائية التي تم الحصول عليها من

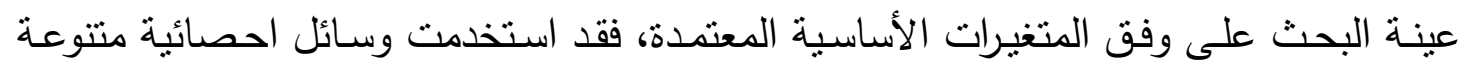
لتحقيق اهداف البحث، قبل الدخول في عرض ومناقشة نتائج البحث قام الباحث باعطاء الإباء فكرة

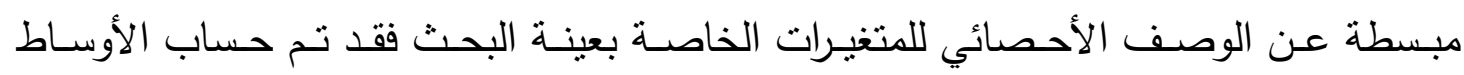
الحسابية والأنحرافات المعيارية لكل من الأختبارات العقلية والمهارية التي تم قياسها وعلى النحو

الآتي:

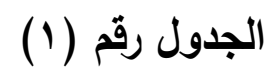

يبين الأوساط الحسابية والأنحرافات المعيارية التي حصلت عليها عينة البحث في اختبارات القدرات العقلية والمهارية

\begin{tabular}{|c|c|c|c|c|c|}
\hline الأنحراف المعياري & الوسط الحسابي & مرحلة الايقاع & والقياس & حصائية & \\
\hline $\mathrm{iv}, \cdot \cdot \mathrm{v}$ & IT,ITV & ايجابي & \multirow{2}{*}{ درجة } & \multirow{2}{*}{ تركيز الأنتباه } & \multirow{2}{*}{ العقلية } \\
\hline $\mid r, r \wedge r$ & rq,orr & سلبي & & & \\
\hline
\end{tabular}

1 نـاجي، قبيس و كامسل ، شـامل : مبـادئ الأحصـاء في التربيـة الرياضـية، جامعـة بغداد، مطبعـة التعليم

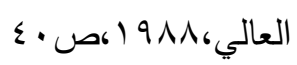




\begin{tabular}{|c|c|c|c|c|c|}
\hline$\cdot, \mathrm{V} \vee \wedge$ & $P V, Q \leq \varepsilon$ & الأيجابية & \multirow{2}{*}{ درجة } & \multirow{2}{*}{ الدفاع عن الملعب } & \multirow{2}{*}{ المهارية } \\
\hline 1,119 & $|9, \varepsilon \wedge|$ & السلبية & & & \\
\hline
\end{tabular}

يتبين في الجدول اعلاه الأوساط الحسابية والاتحرافات المعيارية للقدرات العقلية ( تركيز

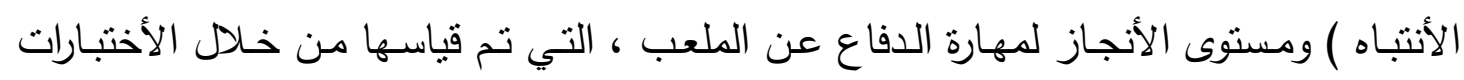

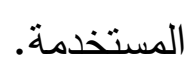

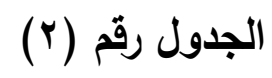

يبين العلاقة بين دورة الإيقاع الحيوي العقلي ( تركيز الأنتباه) للمرحلتين الأيجابية والسلبية

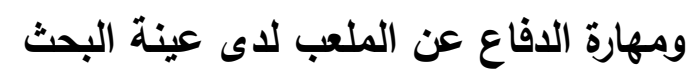

\begin{tabular}{|c|c|c|c|c|}
\hline مستوى الدلالة & قيمة معامل الأرتباط & الدفاع عن الملعب & مرحلة الإيقاع & للمعالجات الاحصائية \\
\hline & \multirow{2}{*}{$\cdot, \wedge \vee \wedge$} & $\cdot, 9 Y 1 *$ & الأيجابية & \multirow{2}{*}{ تركيز الأنتباه } \\
\hline غير معنوي & & $\cdot, 011$ & السلبية & \\
\hline
\end{tabular}

تحت مستوى الدلالة (0. . •) ودرجة الحرية (r) عند ملاحظة الجدول رقم (؟) تبين لنا ان قيمة معامل الأرتباط لمظهر تركيز الأنتباه في

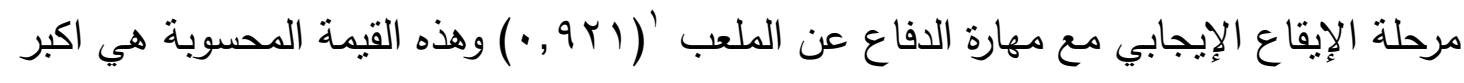

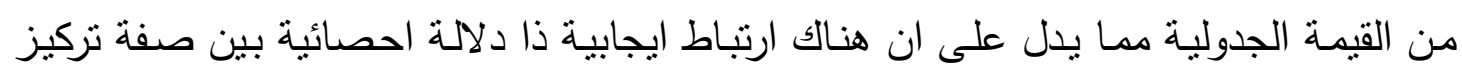
الأنتباه ومهارة الدفاع عن الملعب في مرحلته الايجابية.

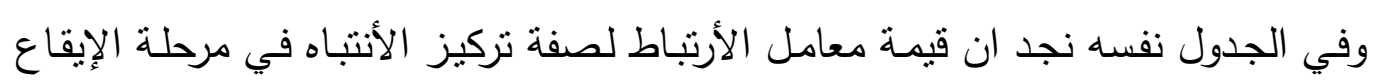

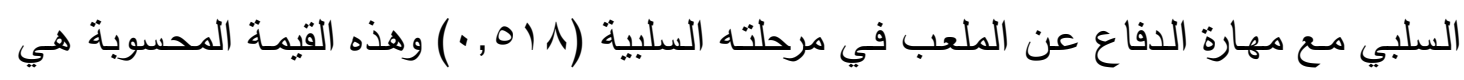

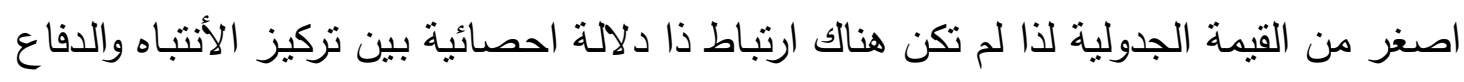
عن الملعب.

\section{ع - r مناقشة ابن النتائج}

من خلال ملاحظة الجدول رقم (Y) نجد ان هناك ارتباط ذا دلالة احصائية في تركيز

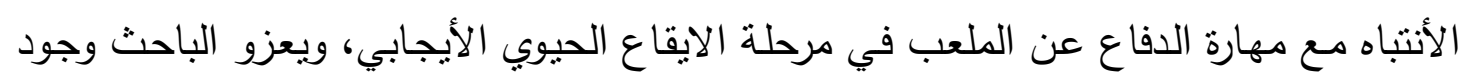

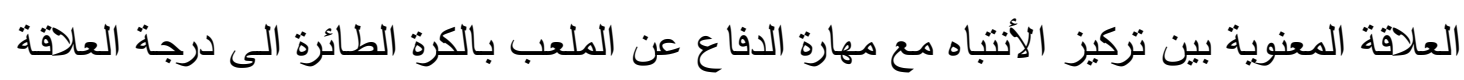

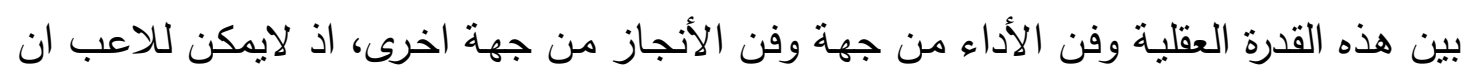

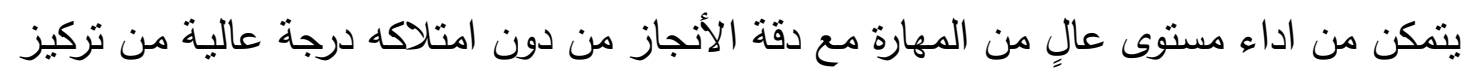


الانتباه، ولعل هذه العلاقة المعنوية هي سبب مهم لتحقيق النتائج المتمييزة للاعب التي تتطلب

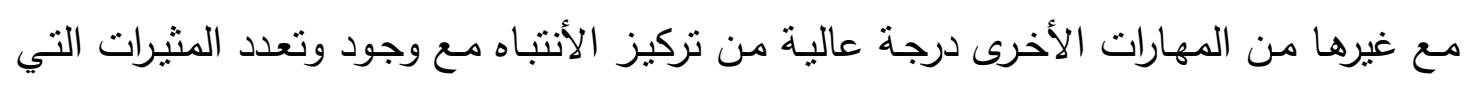
تواجه اللاعب في اثناء ادائه للمهارات وتحقيقه للأنجاز .

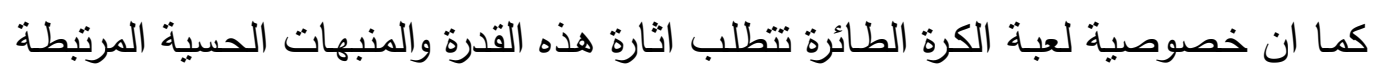
معها عند اداء أي مهارة او محاولة تحقيق أي انجاز على الرغم من اختلاف درجة قوتها وشدتها بما يتتاسب وخصوصية كل مهارة. ويذهب في هذا الرأي ( عبدالستار الضمد) في تحديده لمفهوم تركيز الأنتباه في المجال

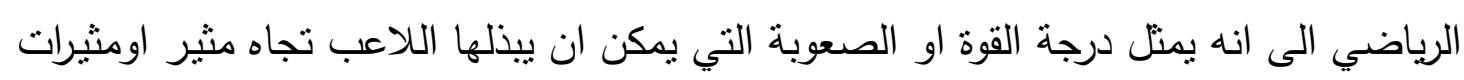

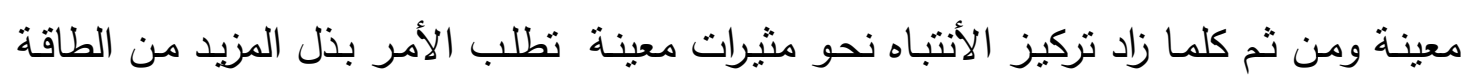
العصبية او العقلية في عملية الأنتباه' وفي متغيرات الدورة العقلية لتركيز الأنتباه في مرحلة الإيقاع الحيوي السلبي لم يظهر ارتباط

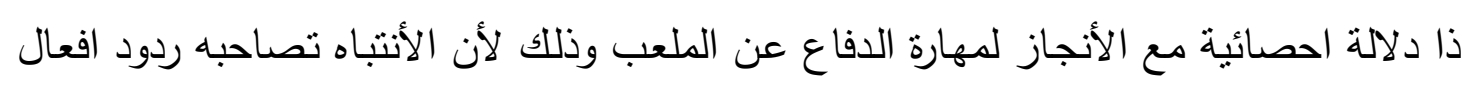

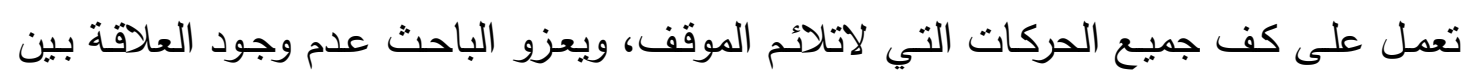

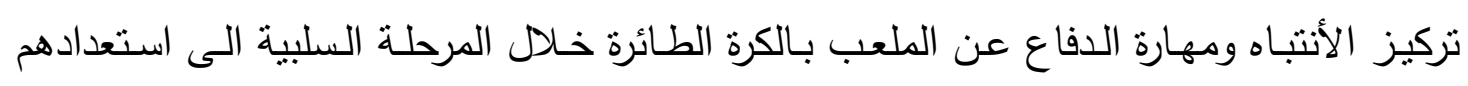

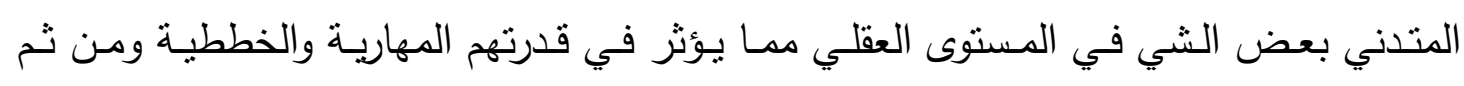
التذبذب في المستوى لتلك القدرات. مما تقدم نجد ان هناك ارتباط معنوي في الإيقاع الحيوي العقلي مع مستوى الأنجاز في

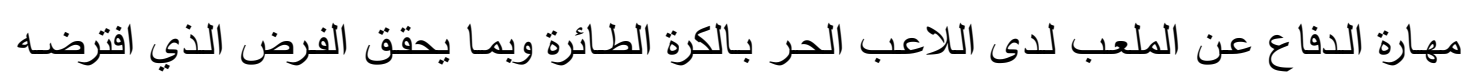
الباحث.

\section{0- الاستتناجات والتوصيات

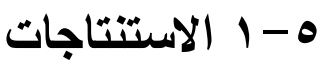

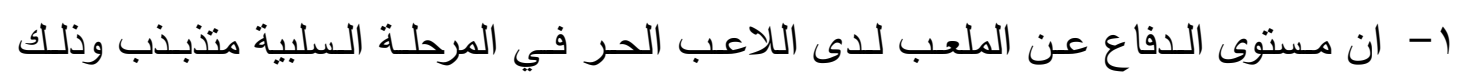
لأستعدادهم المتدني بعض الثنيْ في المستوى العقلي ( نركيز الأنتباه).

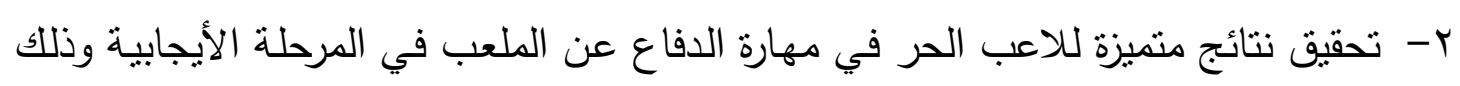
لأمتلاكه درجة عالية من نركيز الأنتباه.

1 1الضمد ،عبدالستار : فسبولوجيا العطليات العقلية، طا، دار الفكر للطباعة والنشر ، عمان،الأردن، Tr. T.... 
r- هناك ارتباط معنوي موجب بين الإيقاع الحيوي العقلي ومهارة الدفاع عن الملعب للى عينة البحث في المرحلة الأيجابية المستخلصة من نتائج الأختبارات. ب-

1- ضرورة مراعاة اللاعبين في اوقات ومراحل الإيقاع الحيوي خاصة الدورة العقلية عند تحديد

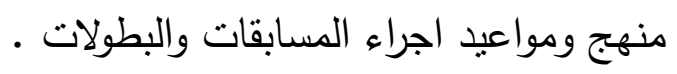

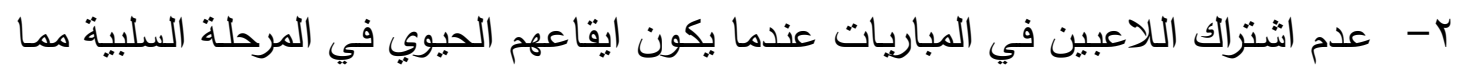

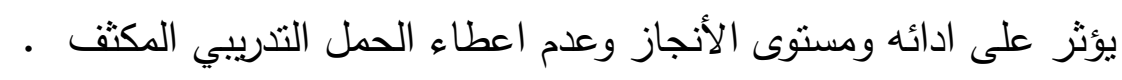

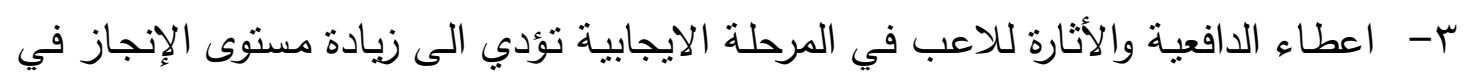

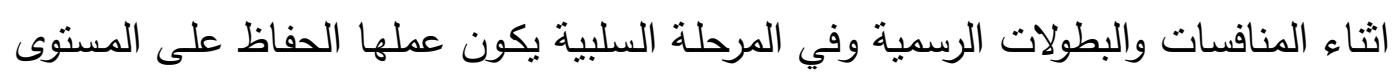

\section{المصادر العربية والأجنبية}

1. احمد، بسطويسى: (999 (199 اسس ونظريات التدريب الرياضي،دار الفكر العربي، القاهرة r. بيك ،علي وعمرصبري:( ـ99 1) الايقاع الحيوي والأنجاز الرياضي ، الأسكندرية.

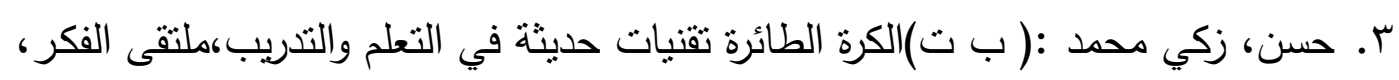

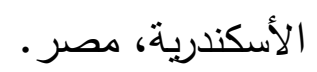

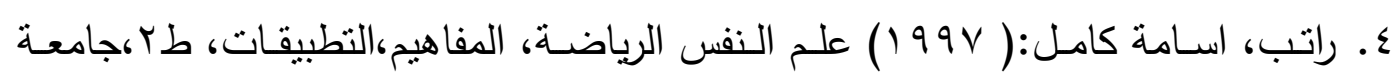
حلوان، القاهرة. 0. الضمد ،عبدالستار : ( . . ب) فسيولوجيا العمليـات العقليـة، طا، دار الفكر للطباعـة والنشر ، عمان، الأردن.

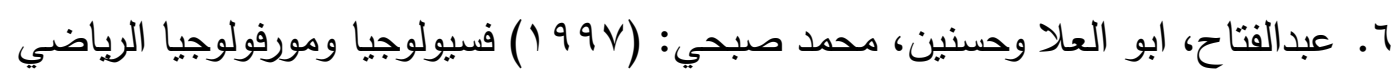

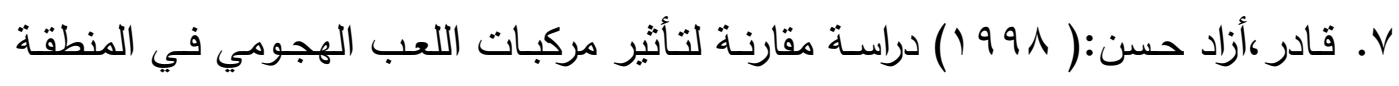

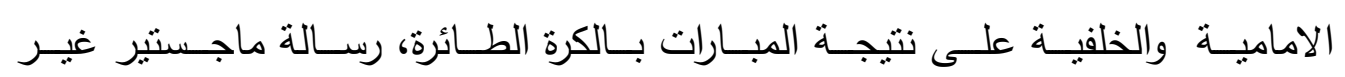
منشورة،جامعة صلاح الدين،كلية التربية الرياضية.

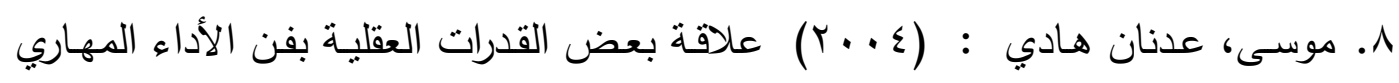

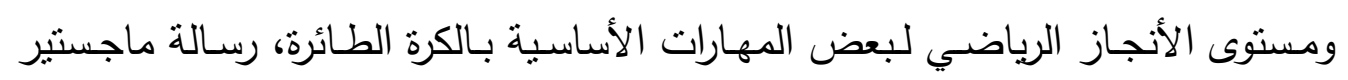
غير منشورة، جامعة الموصل، كليةالتربية الرياضية.

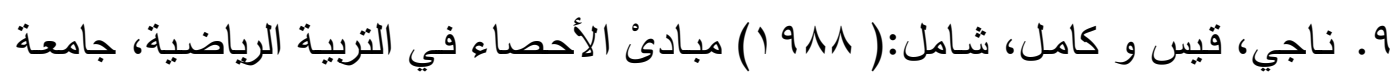
بغداد، مطبعة التعليم العالي.

IV. 
•

$$
\text { الأيوبي الرياض، السعودية. }
$$

11. وطرق القياس والثقويم، دار الفكر العربي، جامعة حلوان، القاهرة.

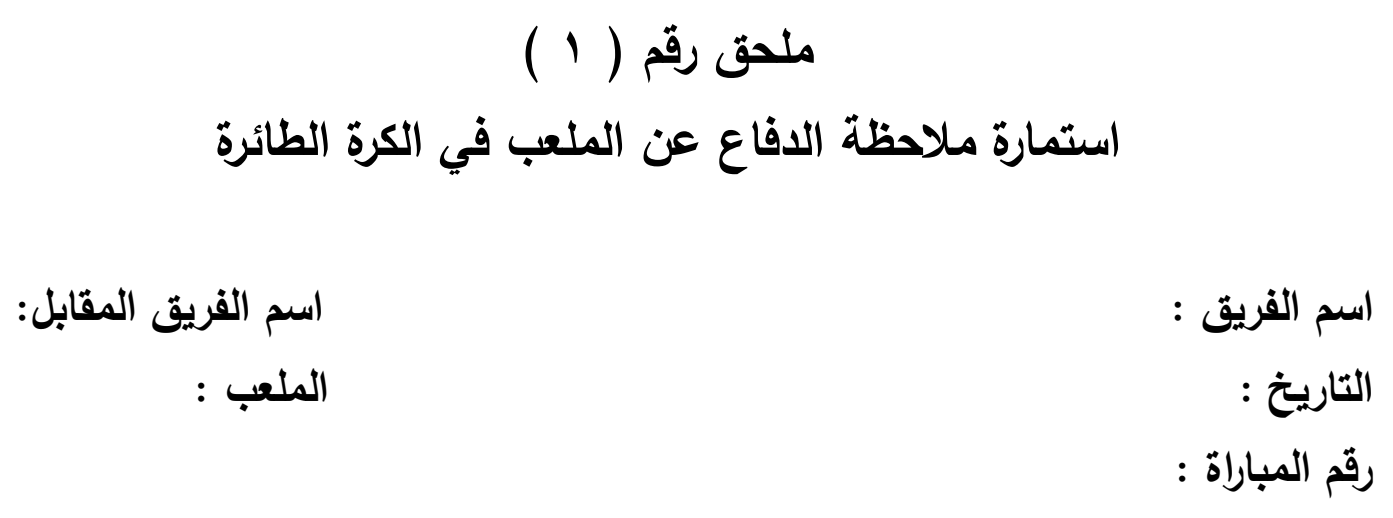

\begin{tabular}{|c|c|c|c|c|c|c|c|c|}
\hline \multirow{2}{*}{$r$} & \multicolumn{3}{|c|}{ الفريق ( } & \multicolumn{2}{|c|}{(} & \multicolumn{2}{|c|}{ الفريق ( } & \multirow{2}{*}{ الأشثواط } \\
\hline & $r$ & 1 & . & $r$ & $r$ & 1 & . & \\
\hline & & & & & & & & 1 \\
\hline & & & & & & & & $r$ \\
\hline & & & & & & & & $r$ \\
\hline & & & & & & & & $\varepsilon$ \\
\hline & & & & & & & & 0 \\
\hline & & & & & & & & المجموع \\
\hline
\end{tabular}

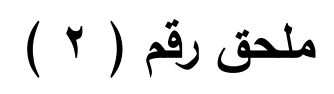

استمارة اختبار بوردون - انفيموف المعدل من قبل عبدالجواد طه لقياس مظاهر

$$
\text { الأنتباه }
$$

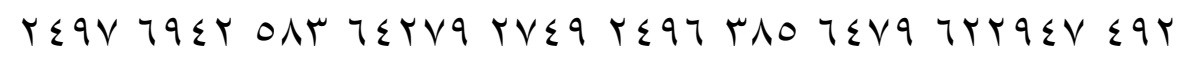

$q r \leq V 7$ OrN $r \leq q 7 \quad r q \leq V$ ONr $T \leq q r \quad r q \leq 7 \quad r q V \leq r \leq q V T V \leq r q$

$r \leqslant q r \quad r \leqslant q 7$ ONr $r \leqslant q \vee q V \leqslant r q \quad r q \leqslant V \quad r \leqslant q r \quad r q \leqslant V \quad r q \leq T \quad r \leqslant q V$

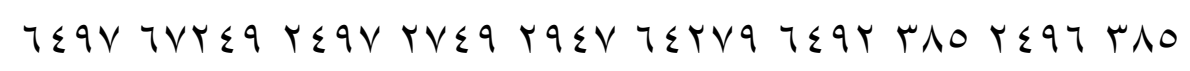

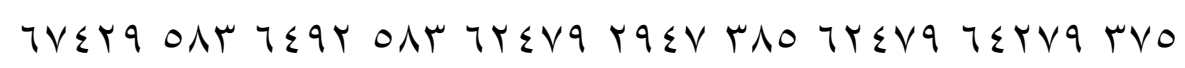

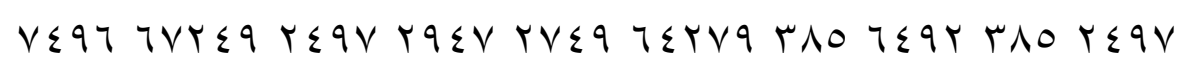




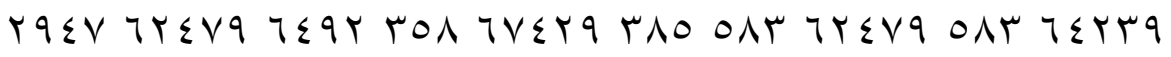

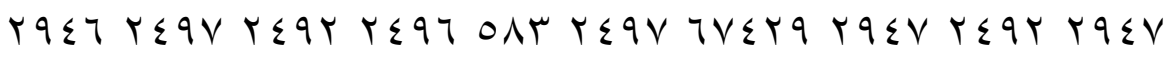

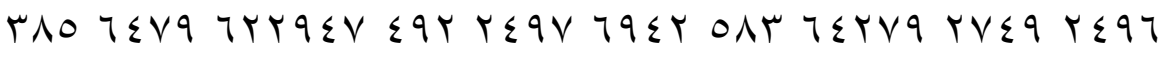

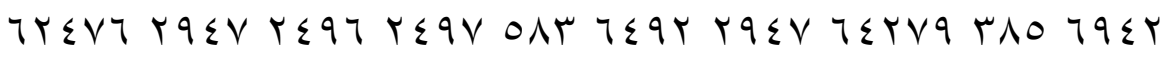

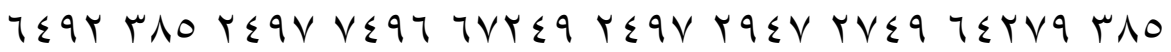

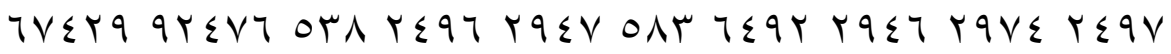
$r \leq q r \quad r \leq q 7$ ONr $r \leq q \vee q V \leq r q \quad r q \leq V \quad r \leq q r \quad r q \leq V \quad r q \leq q \quad r \leq q \vee$

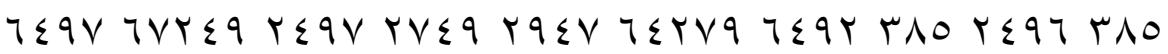

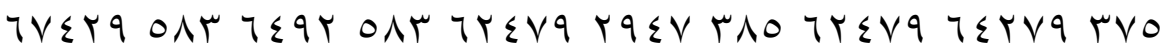

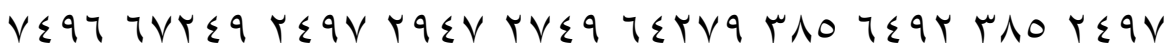

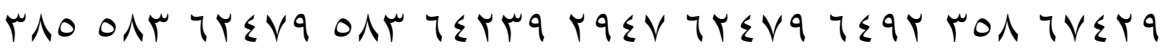

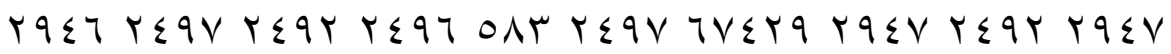

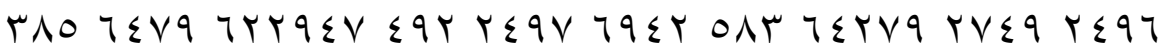

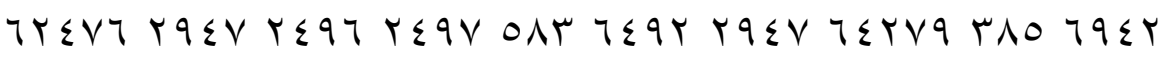

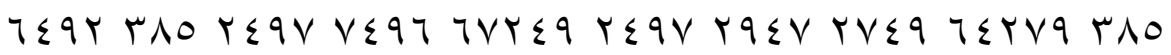

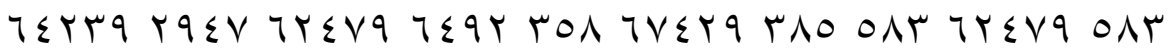

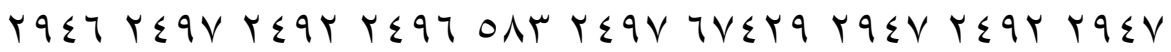

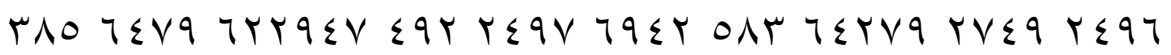

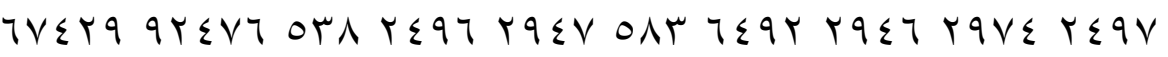

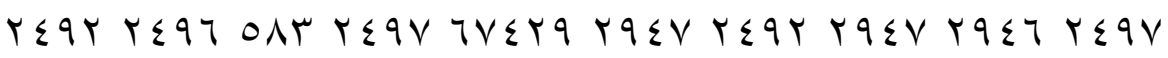

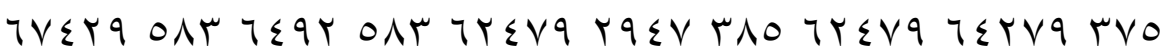

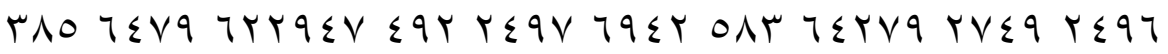

IVY 